\title{
Change in physical activity level and clinical outcomes in older adults with knee pain: a secondary analysis from a randomised controlled trial
}

\author{
Jonathan G. Quicke*, Nadine E. Foster, Peter R. Croft, Reuben O. Ogollah and Melanie A. Holden
}

\begin{abstract}
Background: Exercise interventions improve clinical outcomes of pain and function in adults with knee pain due to osteoarthritis and higher levels of physical activity are associated with lower severity of pain and higher levels of physical functioning in older adults with knee osteoarthritis in cross-sectional studies. However, to date no studies have investigated if change in physical activity level during exercise interventions can explain clinical outcomes of pain and function. This study aimed to investigate if change in physical activity during exercise interventions is associated with future pain and physical function in older adults with knee pain.
\end{abstract}

Methods: Secondary longitudinal data analyses of a three armed exercise intervention randomised controlled trial. Participants were adults with knee pain attributed to osteoarthritis, over the age of 45 years old $(n=514)$ from Primary Care Services in the Midlands and Northwest regions of England. Crude and adjusted associations between absolute change in physical activity from baseline to 3 months (measured by the self-report Physical Activity Scale for the Elderly (PASE)) and i) pain ii) physical function (Western Ontario and McMaster Universities Osteoarthritis Index) and iii) treatment response (OMERACT-OARSI responder criteria) at 3 and 6 months follow-up were investigated using linear and logistic regression.

Results: Change in physical activity level was not associated with future pain, function or treatment response outcomes in crude or adjusted models at 3 or 6 months $(P>0.05)$. A 10 point increase in PASE was not associated with pain $\beta=-0.01(-0.05,0.02)$, physical function $\beta=-0.09(-0.19,0.02)$ or likelihood (odds ratio) of treatment response $1.02(0.99,1.04)$ at 3 months adjusting for sociodemographics, clinical covariates and the trial intervention arm. Findings were similar for 6 month outcome models.

Conclusions: Change in physical activity did not explain future clinical outcomes of pain and function in this study. Other factors may be responsible for clinical improvements following exercise interventions. However, the PASE may not be sufficiently responsive to measure change in physical activity level. We also recommend further investigation into the responsiveness of commonly used physical activity measures.

Trial registration: (ISRCTN93634563). Registered 29th September 2011.

Keywords: Osteoarthritis, Knee, Pain, Physical activity, Exercise, Geriatrics

\footnotetext{
* Correspondence: j.g.quicke@keele.ac.uk

Arthritis Research UK Primary Care Centre, Research Institute for Primary Care

and Health Sciences, Keele University, Keele, Staffordshire ST5 5BG, UK
} 


\section{Background}

Knee pain attributable to osteoarthritis (OA) is both common and disabling in older adults [1]. Exercise and physical activity (PA), including lower limb muscle strengthening and aerobic exercise (for example walking, cycling and swimming) are core recommended treatments in OA clinical guidelines [1-3]. Such interventions are associated with, on average, small to medium effect sizes in terms of reduction in pain and improvements in physical function compared to non-exercise control groups [4-6], although improvements may not be maintained over the longer-term. In order to optimise the effectiveness of exercise interventions, it is important to understand the active components that contribute to improved clinical outcomes [7].

Higher levels of physical activity have been shown to be associated with lower severity of pain and higher physical function in older adults with knee pain in cross-sectional studies [8]. It is plausible that changes in participants' physical activity level as a result of exercise interventions may explain changes in pain and physical functioning either directly or indirectly. For example, increase in levels of moderate and vigorous cardiovascular intensity physical activity are associated with weight loss in longitudinal cohorts with or at risk of knee OA [9] which is associated with reduced pain and improved function in those who are overweight [10]. However, to date, no studies have investigated the association between change in physical activity level and clinical outcomes of pain and function. The aim of this study, therefore, was to investigate if change in physical activity level is associated with future pain, physical function and overall response to treatment [11] in older adults with knee pain.

\section{Methods}

\section{Design}

This study involved secondary analyses of data from a multi-centre, pragmatic, three-parallel group, randomised controlled trial (RCT) of three physical therapistled exercise interventions (The Benefits of Effective Exercise for knee Pain-BEEP trial ISRCTN 93634563) [12]. Longitudinal data from all three groups at baseline, three months (following treatment in two of three groups) and six months (following treatment in all groups) were combined, with a priori adjustment for intervention group allocation. Ethical approval was provided by the North West 1 Research Ethics Committee, Cheshire, UK (REC reference 10/H1017/45). Full detail of the RCT is available elsewhere [12] but a concise summary is provided below for context.

\section{Participants}

Participants were adults with knee pain attributable to $\mathrm{OA}$ from the BEEP trial $(n=514)$. Inclusion criteria were a clinical diagnosis of knee OA made by either a general practitioner or a primary care research nurse based on age (being 45 years old or older), the presence of pain and/or stiffness in one or both knees [1] and the exclusion of knee pain due to other sources, such as those who had pain due to trauma or recent injury, potentially serious pathology other than OA (such as malignancy or rheumatoid arthritis) and those who had undergone previous total knee replacements. Those with exercise contraindications (such as those with unstable cardiovascular disorders, severe hypertension or congestive heart failure) and those unable to travel to physical therapy treatment centres were also excluded [12].

Participants were recruited from 65 general practices in the midlands and northwest regions of England from: i) records of general practitioner consulters with knee pain in the last year, ii) those referred to physical therapy with knee pain and, iii) adults registered at participating general practices who responded to a questionnaire and reported knee pain [12].

\section{Trial intervention arms}

The three physical therapist-delivered exercise intervention arms were: usual care (UC), individually tailored exercise (ITE) and targeted exercise adherence (TEA). All participants received an advice and information booklet in addition to an exercise programme delivered one-toone for up to three (UC and ITE) and six months (TEA). Additional intervention detail is provided in Additional file 1: Table S1.

\section{Outcomes}

\section{Pain and physical function}

Knee pain severity and physical function were assessed using the Western Ontario and McMaster Universities Osteoarthritis Index (WOMAC) pain and function subscales [13]. The pain subscale comprises five items measuring self-reported pain during various activities and gives a total score ranging from 0 (no pain) to 20 (maximum pain). The physical function subscale comprises 17 items and measures the self-reported difficulty an individual has with a broad range of physical functions giving a total score ranging from 0 (no disability) to 68 (maximum disability). Both subscales have been widely used in knee OA studies and their clinimetric properties have been widely investigated elsewhere [13-15].

\section{Treatment response}

Clinically important response to treatment was assessed using the Outcome Measures in Rheumatology Clinical Trials clinical responder criteria (OMERACT-OARSI responder criteria) [11, 16]. This internationally agreed measure combines outcome data on pain and physical function from the WOMAC scales with patient's global 
assessment of change (recorded using a 6 point Likert Scale) $[11,16]$. Treatment responders are classified as those who either make a large improvement in pain or function ( $\geq 50 \%$ improvement and absolute change $\geq 20$ in a $0-100$ scale) or a small improvement ( $\geq 20 \%$ improvement and absolute change $\geq 10$ in a $0-100$ scale) in two out of three of pain, physical function or global assessment of change (at follow-up compared to baseline).

\section{Determinants}

\section{Change in physical activity}

Absolute change in physical activity from baseline to three month follow-up (referred to henceforth as "change in physical activity") was calculated by subtracting baseline physical activity score from follow-up physical activity score at three months using the self-report Physical Activity Scale for the Elderly (PASE) [17]. The PASE scale (scored from 0 to over 400 with higher scores indicating higher levels of physical activity) measures physical activity in the previous week summed from questions regarding the frequency and duration of household, leisure time and work physical activity. It has demonstrated construct validity in terms of correlation with 6 min walk test $(r=0.35)$ and knee strength $(0.41)$ in older adults with knee pain [18] and test-retest reliability in older adults (intra-class correlation 0.75) [17]. The PASE scale has been used in previous longitudinal studies of knee pain and OA [19-21].

\section{Potential confounders}

A range of sociodemographic and clinical covariates measured within the BEEP dataset may be potential confounders of the relationship between change in physical activity and clinical outcomes due to their association with both physical activity [8] and clinical outcome [22, 23]. These included age, gender, Body Mass Index (BMI), individual socioeconomic status [24], employment status, comorbidities (categorised into none, one and two or more), depression measured by the Personal Health Questionnaire (PHQ 8) [25], anxiety measured by the generalized Anxiety Disorder Questionnaire (GAD-7) [26], and widespread pain measured by the Manchester Widespread Pain criteria [27].

\section{Analyses}

All analyses were carried out using STATA version 13.1 [28] and all primary longitudinal analyses utilised a multiply imputed dataset. Multiple imputation (25 imputations) using chained equations was used to adjust for the effect of missing data by maximising sample size and reducing the possible bias associated with loss to followup and missing data [29] since there was between 12 and $22 \%$ missing clinical outcome data at 3 and 6 months. A wide range of sociodemographic and clinical variables available within the BEEP dataset, including the outcome variables, were used in the imputation model [30].

\section{Descriptive statistics}

Baseline characteristics (complete case) together with longitudinal descriptive statistics (at baseline, three and six months) of physical activity, change in physical activity (measured by the PASE) and clinical outcomes of pain, physical function (WOMAC) and treatment response (OMERACT-OARSI responder criteria) were summarised using means and standard deviations (SD) or frequency and percentage as appropriate.

\section{Analyses to investigate the association between change in physical activity and clinical outcome}

Associations between change in physical activity and clinical outcomes of pain, physical function and treatment response at three and six months were determined using linear and logistic regression. Both univariable and multivariable models were fitted. ANCOVA type multivariable models were used where the clinical outcome variable of interest (pain or function) at 6 months (and subsequently 12 months) were adjusted for baseline clinical severity (pain in the pain and treatment response models, and function in the function model), potential confounders and the trial intervention arm. The a priori decision to hold the intervention arm within multivariable models was made to account for any between trial arm treatment effects (although similar clinical improvements were found in all groups). Additional methodological detail regarding adjusted model building is provided in the Additional file 2.

\section{Sensitivity analyses}

Sensitivity analyses on missing data was performed by use of complete case analysis (CCA), which restricts the analysis to subjects with complete data on the variables involved in the analysis. CCA assumes that missing cases are missing completely at random and makes the assumption that results would be similar to intended sample results [31].

\section{Results}

\section{Descriptive statistics}

Table 1 describes the baseline characteristics of the 514 BEEP trial participants. The sample was $51 \%$ female with a participant mean (SD) age of 62.8 (9.7) years. Mean WOMAC pain score (SD) was 8.4 (3.5) and physical function score was 28.1 (12.2) suggesting that on average participants had moderate levels of clinical severity. Mean physical activity score, measured by the PASE, was 177 (83.3) (see Table 2). 
Table 1 Baseline characteristics

\begin{tabular}{|c|c|}
\hline Characteristic & Total $(n=514)$ \\
\hline \multicolumn{2}{|l|}{ Age, $n(\%)$, years } \\
\hline $45-49$ & $52(10)$ \\
\hline $50-59$ & $153(30)$ \\
\hline $60-69$ & $183(36)$ \\
\hline $70-79$ & $99(19)$ \\
\hline$\geq 80$ & $27(5)$ \\
\hline Female, $n(\%)$ & $262(51)$ \\
\hline \multicolumn{2}{|l|}{$\mathrm{BMI}, n(\%),{ }^{*}$} \\
\hline Underweight/ normal & $97(20)$ \\
\hline Overweight & $208(42)$ \\
\hline Obese & $192(39)$ \\
\hline Currently employed n (\%)* & $214(42)$ \\
\hline \multicolumn{2}{|c|}{ Socioeconomic category, $n(\%){ }^{*}$} \\
\hline Professional & $166(43)$ \\
\hline Intermediate & $94(25)$ \\
\hline Routine and manual work & $124(32)$ \\
\hline \multicolumn{2}{|l|}{ Comorbidities, n (\%) } \\
\hline None & $164(32)$ \\
\hline 1 comorbidity & $180(35)$ \\
\hline 2 or more comorbidities & $170(33)$ \\
\hline PHQ 8, 0-24, mean (SD) * & $4.0(+/-4.7)$ \\
\hline \multicolumn{2}{|l|}{ WOMAC, mean (SD) } \\
\hline Pain, $0-20, *$ & $8.4(+/-3.5)$ \\
\hline Function, $0-68, *$ & $28.1(+/-12.3)$ \\
\hline Stiffness, $0-8, *$ & $3.7(+/-1.7)$ \\
\hline \multicolumn{2}{|c|}{ Knee pain duration, $n(\%)$, years * } \\
\hline$\leq 1$ & $125(25)$ \\
\hline More than 1 but $<5$ & $198(39)$ \\
\hline More than 5 but $<10$ & $94(19)$ \\
\hline $10+$ & $91(18)$ \\
\hline Widespread pain $n(\%){ }^{*}$ & $79(15)$ \\
\hline
\end{tabular}

Footnote: Baseline descriptive statistics based on complete cases; ${ }^{*}=$ subject to missing data (hence individual item frequencies may not add to total sample). Missing data was $2 \%$ in primary clinical variables, less than $10 \%$ in all remaining variables except socioeconomic category which was $25 \%$ missing. Body Mass Index: less than $25=$ underweight/ normal, 25 or more but less than $30=$ overweight, 30 or more $=$ obese. Comorbidities included (in descending order of frequency) Hypertension, Asthma, Diabetes, Angina, Heart attack and Heart failure

Abbreviations: BMI Body Mass Index, PHQ 8 Personal Health Depression Questionnaire (higher scores indicate lower mood), SD Standard deviation; Widespread pain = Manchester Widespread Pain [27]; WOMAC Western Ontario and McMaster Universities Osteoarthritis Index

The proportion of the baseline sample lost to follow up was $17 \%$ at three months and $11 \%$ at six months. For information, participants lost to follow-up had slightly worse pain and function at baseline, higher levels of depression and anxiety at baseline and were less likely to have used facilities for physical activity in the last 7 days [30]. Mean
Table 2 Physical activity and clinical outcome longitudinal summary statistics

\begin{tabular}{llll}
\hline Variables (range) & Baseline & 3 months & 6 months \\
\hline PASE (0-400+) & $177.0(83.3)$ & $192.1(87.9)$ & $190.5(89.3)$ \\
WOMAC pain (0-20) & $8.4(3.5)$ & $6.7(3.6)$ & $6.3(3.9)$ \\
WOMAC function (0-68) & $28.1(12.2)$ & $23.6(12.5)$ & $21.7(13.7)$ \\
OMERACT-OARSI response (\%) & NA & 45 & 52 \\
\hline
\end{tabular}

Footnote: Multiple imputed data (combined results from 25 imputed datasets). All values are mean scores (standard deviation) except OMERACT-OARSI response which are given in percentages. All scores indicate higher levels of the variable except WOMAC function with higher scores indicating lower functioning Abbreviations: OMERACT-OARSI Outcome Measures in Rheumatology Clinical Trials-Osteoarthritis Research Society International, PASE Physical Activity Scale for the Elderly, WOMAC Western Ontario and McMaster Universities Osteoarthritis Index

PASE increased to 192.1 (87.9) at three month follow-up and then remained relatively stable at six month follow-up 190.5 (89.3). Clinical outcome scores of pain and function improved over time with $45 \%$ classified as treatment responders at three months and $52 \%$ at six months follow-up. At three months, mean WOMAC pain and function were 6.7 (3.6) and 23.6 (12.5) respectively, changing to 6.3 (3.9) and 21.7 (13.7) at six month follow-up (see Table 2).

The mean PASE change score between baseline and three months was an increase of 15.1 with substantial individual variation as indicated by a standard deviation of 87.4. Change scores for physical activity and clinical outcomes are reported in Table 3.

\section{Associations between change in physical activity and clinical outcome}

Univariable models showed change in physical activity was not significantly associated with any of the three clinical outcomes of pain, physical function (Tables 4 and 5) or treatment response at both three and six months $(p>0.05)$ (Table 6).

Change in physical activity remained non-significantly associated with clinical outcomes in all multivariable clinical outcome models adjusting for age, continuous BMI, pain duration, depression, the trial intervention

Table 3 Physical activity, pain and function change scores

\begin{tabular}{lll}
\hline Change variable & $\begin{array}{l}\text { Mean score baseline } \\
\text { to } 3 \text { months(SD) }\end{array}$ & $\begin{array}{l}\text { Mean score baseline } \\
\text { to } 6 \text { months(SD) }\end{array}$ \\
\hline Change in PASE & $15.1(87.4)$ & $13.5(86.9)$ \\
Change in WOMAC pain & $-1.6(3.2)$ & $-2.1(3.5)$ \\
Change in WOMAC function & $-4.5(10.1)$ & $-6.4(11.8)$
\end{tabular}

Footnote: Multiple imputed data (combined results from 25 imputed datasets); all change scores calculated by subtracting the score at baseline from the score at three or six month follow up; Higher change in PASE scores indicate higher physical activity at follow up compared to baseline; Negative change in WOMAC pain and function scores indicate reduced pain and higher function at follow-up compared to baseline

Abbreviations: PASE Physical Activity Scale for the Elderly, WOMAC Western Ontario and McMaster Universities Osteoarthritis Index 
Table 4 The association between change in physical activity level and pain and function at three months follow-up

\begin{tabular}{|c|c|c|c|c|}
\hline & \multicolumn{2}{|c|}{ WOMAC pain at 3 months } & \multicolumn{2}{|c|}{ WOMAC function at 3 months } \\
\hline & $\begin{array}{c}\text { Unadjusted } \\
\beta(95 \% \mathrm{Cl})\end{array}$ & $\begin{array}{c}\text { Adjusted } \\
\beta(95 \% \mathrm{Cl})\end{array}$ & $\begin{array}{c}\text { Unadjusted } \\
\beta(95 \% \mathrm{Cl})\end{array}$ & $\begin{array}{l}\text { Adjusted } \\
\beta(95 \% \mathrm{Cl}) \\
\end{array}$ \\
\hline \multicolumn{5}{|l|}{ Change in physical activity } \\
\hline PASE 10 point increase & $0.01(-0.03,0.05)$ & $-0.01(-0.05,0.02)$ & $0.00(-0.14,0.15)$ & $-0.09(-0.19,0.02)$ \\
\hline \multicolumn{5}{|l|}{ Potential confounders } \\
\hline Age & $0.06^{* *}(0.02,0.09)$ & $0.05^{\star *}(0.03,0.08)$ & $0.23^{\star *}(0.12,0.35)$ & $0.15^{\star *}(0.06,0.24)$ \\
\hline Continuous BMI & $0.13^{* *}(0.08,0.19)$ & $0.06^{*}(0.01,0.10)$ & $0.55^{\star \star}(0.35,0.74)$ & $0.21^{*}(0.05,0.37)$ \\
\hline WOMAC pain baseline & $0.60^{* *}(0.52,0.67)$ & $0.52^{* *}(0.44,0.60)$ & $1.93^{\star *}(1.66,2.21)$ & \\
\hline $\begin{array}{l}\text { WOMAC function baseline } \\
\text { Pain duration } \\
\text { (reference }<1 \text { year) }\end{array}$ & $0.16^{\star *}(0.14,0.18)$ & & $0.68^{* *}(0.61,0.75)$ & $0.60^{* *}(0.53,0.68)$ \\
\hline$>1$ year and $<5$ years & $1.13^{* *}(0.31,1.94)$ & $1.06^{\star *}(0.39,1.72)$ & $3.26^{*}(0.34,6.18)$ & $2.67^{\star}(0.40,4.94)$ \\
\hline$>5$ years and $<10$ years & $2.09^{* *}(1.11,3.07)$ & $1.60^{* *}(0.80,2.41)$ & $5.64^{* *}(2.20,9.08)$ & $3.82^{* *}(1.15,6.50)$ \\
\hline$>10$ years & $2.07^{* *}(1.07,3.06)$ & $1.48^{* *}(0.66,2.31)$ & $4.93^{* *}(1.46,8.01)$ & $3.42^{*}(0.69,6.15)$ \\
\hline $\begin{array}{l}\text { PHQ8 depression } \\
\text { Intervention arm } \\
\text { (reference usual physio) }\end{array}$ & $0.19^{* *}(0.12,0.26)$ & $0.11^{\star *}(0.05-0.17)$ & $0.68^{* *}(0.45,0.91)$ & $0.23^{*}(0.03,0.42)$ \\
\hline Individually tailored exercise & $0.26(-0.53,1.05)$ & $0.04(-0.59,0.67)$ & $-0.04(-2.82,-2.74)$ & $-0.38(-2.50,1.74)$ \\
\hline Targeted exercise adherence & $0.27(-0.52,1.06)$ & $0.12(-0.50,0.75)$ & $1.56(-1.21,4.33)$ & $0.47(-1.63,2.57)$ \\
\hline
\end{tabular}

Key: White $=$ Unadjusted Models; Blue $=$ Adjusted pain at 3 months model; Gold = Adjusted physical function at 3 months model

Footnotes: Multiple imputed data; multiple linear regression adjusted models selected via backwards elimination holding treatment arm and change in physical activity in the model. * $=$ statistically significant $\beta$ coefficient $P<0.05{ }^{* *}=$ statistically significant $\beta$ coefficient $P<0.01$. Higher WOMAC scores indicate higher pain and worse function. Higher PASE score indicates higher level of physical activity, " absolute change in PASE calculated by subtracting the baseline score from the score at three months. Higher PHQ8 depression scores indicate worse depression

Abbreviations: $\beta$ Unstandardized coefficients, BMI Body Mass Index; $\mathrm{Cl}=$ Confidence Interval, PASE Physical Activity Scale for the Elderly, PHQ8 Personal Health Questionnaire, WOMAC Western Ontario and McMaster Osteoarthritis Index

arm and baseline pain/function $(P>0.05)$ (see Tables 4 , 5 and 6). An increase of 10 PASE points between baseline and three months had a non-statistically significant, adjusted association with WOMAC pain at three $(\beta=-$ 0.01 (95\% Confidence Interval $-0.05,0.02))$ and six $(\beta=$ $-0.02(-0.06,0.02))$ months. Interpreting these best estimate $\beta$ coefficients, for every increase in physical activity of ten points on the PASE, WOMAC pain score decreased by 0.01 at three months and 0.02 at six months. These results are not statistically significant since the 95\% confidence intervals cross zero. Similarly, an increase of 10 points on the PASE scale

Table 5 The association between change in physical activity level and pain and function at six months follow-up

\begin{tabular}{|c|c|c|c|c|}
\hline & \multicolumn{2}{|c|}{ WOMAC pain at 6 months } & \multicolumn{2}{|c|}{ WOMAC function at 6 months } \\
\hline & $\begin{array}{c}\text { Unadjusted } \\
\beta(95 \% \mathrm{Cl})\end{array}$ & $\begin{array}{l}\text { Adjusted } \\
\beta(95 \% \mathrm{Cl})\end{array}$ & $\begin{array}{c}\text { Unadjusted } \\
\beta(95 \% \mathrm{Cl})\end{array}$ & $\begin{array}{c}\text { Adjusted } \\
\beta(95 \% \mathrm{Cl})\end{array}$ \\
\hline $\begin{array}{l}\text { Change in Physical activity } \\
\text { PASE } 10 \text { point increase" } \\
\text { Potential confounders }\end{array}$ & $-0.00(-0.05,0.04)$ & $-0.02(-0.06,0.02)$ & $0.00(-0.16,0.17)$ & $-0.09(-0.22,0.04)$ \\
\hline $\begin{array}{l}\text { Age } \\
\text { WOMAC pain baseline } \\
\text { WOMAC function baseline } \\
\text { Pain duration } \\
\text { (reference }<1 \text { year) }\end{array}$ & $\begin{array}{c}0.06^{* *}(0.03,0.10) \\
0.64^{* *}(0.56,0.73) \\
0.17^{* *}(0.015,0.20)\end{array}$ & $\begin{array}{l}0.05^{\star *}(0.02-0.08) \\
0.59^{* *}(0.51,0.67)\end{array}$ & $\begin{array}{l}0.28^{* *}(0.16,0.40) \\
1.97^{* *}(1.66,2.27) \\
0.66^{\star *}(0.57,0.74)\end{array}$ & $\begin{array}{l}0.19^{* *}(0.09,0.29) \\
0.59^{* *}(0.50,0.67)\end{array}$ \\
\hline$>1$ year and $<5$ years & $1.08^{*}(0.19,1.97)$ & $0.96^{*}(0.22,1.69)$ & $4.22^{* *}(1.15,7.30)$ & $3.53^{\star *}(1.01,6.05)$ \\
\hline$>5$ years and $<10$ years & $2.57^{\star \star}(1.52,3.62)$ & $2.03^{* *}(1.17,2.90)$ & $8.50^{\star *}(4.83,12.17)$ & $6.65^{\star *}(3.63,9.67)$ \\
\hline$>10$ years & $2.17^{\star *}(1.09,3.25)$ & $1.46^{\star *}(0.56,2.35)$ & $6.31^{* *}(2.61,10.01)$ & $4.57^{\star *}(1.52,7.62)$ \\
\hline $\begin{array}{l}\text { PHQ8 depression } \\
\text { Intervention arm } \\
\text { (reference usual physio) }\end{array}$ & $0.18^{* *}(0.10,0.25)$ & $0.10^{* *}(0.04,0.16)$ & $0.73^{* *}(0.48,0.98)$ & $0.36^{\star *}(0.14,0.58)$ \\
\hline Individually tailored exercise & $0.02(-0.86,0.90)$ & $-0.25(-0.96,0.47)$ & $0.86(-2.14,3.87)$ & $0.51(-1.89,2.90)$ \\
\hline Targeted exercise adherence & $-0.12(-0.98,0.74)$ & $-0.26(-0.95,0.44)$ & $0.08(-2.93,3.09)$ & $-0.72(-3.13,1.69)$ \\
\hline
\end{tabular}

Key: White = Unadjusted Models; Blue = Adjusted pain at 6 months model; Gold = Adjusted physical function at 6 months model

Footnotes: Multiple imputed data, multiple linear regression adjusted models selected via backwards elimination holding treatment arm and change in physical activity in the model. Regression coefficients shown are rounded to two decimal places and a score of -0.00 is used to indicate a very small yet negative confidence interval coefficient. ${ }^{*}=$ statistically significant $\beta$ coefficient $P<0.05 ; * *=$ statistically significant $\beta$ coefficient $P<0.01$. Higher WOMAC scores indicate higher pain and worse function. Higher PASE score indicates higher level of physical activity, " absolute change in PASE calculated by subtracting the baseline score from the score at three months. Higher PHQ8 depression scores indicate worse depression

Abbreviations: $\beta$ Unstandardized coefficients, BMI Body Mass Index, CI Confidence Interval, PASE Physical Activity Scale for the Elderly, PHQ8 = Personal Health Questionnaire, WOMAC Western Ontario and McMaster Osteoarthritis Index 
Table 6 The association between change in physical activity level and treatment response at three and six months follow-up

\begin{tabular}{|c|c|c|c|c|}
\hline & \multicolumn{2}{|c|}{ OMERACT OARSI response at 3 months } & \multicolumn{2}{|c|}{ OMERACT OARSI response at 6 months } \\
\hline & $\begin{array}{l}\text { Unadjusted } \\
\text { OR }(95 \% \mathrm{Cl})\end{array}$ & $\begin{array}{c}\text { Adjusted Model } \\
\text { OR }(95 \% \mathrm{Cl})\end{array}$ & $\begin{array}{l}\text { Unadjusted } \\
\text { OR }(95 \% \mathrm{Cl})\end{array}$ & Adjusted Model \\
\hline \multicolumn{5}{|l|}{ Change in PA } \\
\hline PASE 10 point increase ${ }^{\#}$ & $1.01(0.99,1.04)$ & $1.02(0.99,1.04)$ & $1.01(0.98,1.03)$ & $1.01(0.98,1.04)$ \\
\hline \multicolumn{5}{|l|}{ Potential confounders } \\
\hline WOMAC pain baseline & $1.11^{* *}(1.05,1.18)$ & $1.15^{\star \star}(1.08,1.23)$ & $1.08^{* *}(1.03,1.14)$ & $1.12^{\star \star}(1.06,1.19)$ \\
\hline $\begin{array}{l}\text { Age } \\
\text { Pain duration } \\
\text { (reference }<1 \text { year) }\end{array}$ & $0.97^{\star *}(0.95,0.99)$ & $0.97^{\star *}(0.94,0.99)$ & $0.98^{*}(0.96,1.00)$ & $0.97^{* *}(0.95,0.99)$ \\
\hline$>1$ year and $<5$ years & $0.49^{\star *}(0.30,0.80)$ & $0.46^{* *}(0.28,0.78)$ & $0.57^{*}(0.35,0.93)$ & $0.56^{\star}(0.34,0.93)$ \\
\hline$>5$ years and $<10$ years & $0.36^{\star *}(0.20,0.66)$ & $0.31^{\star *}(0.17,0.59)$ & $0.30^{\star *}(0.17,0.54)$ & $0.27^{\star *}(0.15,0.50)$ \\
\hline$>10$ years & $0.64(0.35,1.17)$ & $0.59(0.32,1.10)$ & $0.45^{\star \star}(0.25,0.80)$ & $0.41^{\star *}(0.22,0.74)$ \\
\hline $\begin{array}{l}\text { PHQ8 depression } \\
\text { Intervention arm } \\
\text { (reference usual physio) }\end{array}$ & $0.98(0.95,1.02)$ & $0.95^{*}(0.91,0.99)$ & $0.98(0.94,1.02)$ & $0.95^{\star}(0.91,0.99)$ \\
\hline Individually tailored exercise & $1.08(0.70,1.68)$ & $1.05(0.66,1.68)$ & $1.06(0.68,1.66)$ & $1.04(0.65,1.67)$ \\
\hline Targeted exercise adherence & $1.02(0.64,1.62)$ & $0.95(0.58,1.56)$ & $1.22(0.78,1.92)$ & $1.15(0.71,1.86)$ \\
\hline
\end{tabular}

Key: White = Unadjusted Models; Blue = Adjusted OMERACT-OARSI treatment response models

Footnotes: Multiple imputed data, multiple logistic regression adjusted models selected via backwards elimination holding treatment arm and change in physical activity in the model. Higher WOMAC scores indicate higher pain. ${ }^{*}=$ statistically significant OR $\mathrm{P}<0.05$; ${ }^{* *}=$ statistically significant OR P $<0.01$. Higher PASE score indicates higher level of physical activity, " absolute change in PASE calculated by subtracting the baseline score from the score at three months. Higher PHQ8 depression scores indicate worse depression

Abbreviations: $\beta$ Unstandardized coefficients, CI Confidence Interval, OMERACT OARSI Osteoarthritis Research Society International set of responder criteria for osteoarthritis clinical trials, PASE Physical Activity Scale for the Elderly, PHQ8 Personal Health Questionnaire, WOMAC Western Ontario and McMaster Osteoarthritis Index; yr. = year

had a non-statistically significant adjusted association with function at three $\beta=-0.09(-0.19,0.02)$ and six months $\beta=-0.09(-0.22,0.04)$ and, treatment response at three months $\mathrm{OR}=1.02(0.99,1.04)$ and six months $\mathrm{OR}=1.01(0.98,1.04)$. Interpreting the treatment response best estimate odds ratios, for every increase in physical activity of ten points on the PASE, participants had a $2 \%$ increase in the odds of being able to be classified as a treatment responder using the OMERACT-OARSI criteria at three month follow-up and $1 \%$ increase in odds at six month follow-up but these findings were not statistically significant since the $95 \%$ confidence interval odds ratios cross one.

Complete case sensitivity analyses, investigating the adjusted association between a 10 point increase in PASE and clinical outcomes of WOMAC pain and function at 3 and 6 months produced similar non-statistically significant associations (results not shown).

\section{Discussion}

\section{Main findings}

This study investigated whether change in physical activity was associated with future clinical outcomes of pain and physical function in older adults with knee pain attributable to OA. This question is novel and important since change in physical activity may explain clinical improvements following exercise interventions and inform future interventions. The main finding from this RCT was that change in physical activity level was not associated with future pain, physical functioning or treatment response at either three or six month follow-up. Small $\beta$ coefficients were expected given the difference in scale between the PASE $(0-400+)$ and WOMAC pain and function scores (0-20 and 0-68 respectively) (since the PASE scale is larger by approximately a factor of 20 than the WOMAC pain scale). However, even taking this in to account and allowing for a 10 point change in PASE, the magnitude of associations were very small, nonstatistically significant and do not appear to be of clinical importance (Tables 4, 5 and 6). For example, extrapolating from the $\beta$ coefficients, changing physical activity by a full standard deviation (83 points on the PASE) would be associated with less than a 1 point change in WOMAC pain or function at either three or six months. Similarly, large changes in physical activity would only have a very small effect on the odds of being an OMERACT-OARSI responder.

The null association findings (Tables 4, 5 and 6) suggest that change in overall general physical activity level, as measured by the PASE, does not explain clinical outcome following exercise intervention within the BEEP trial and that other variables may be responsible for the observed improvements in pain and function (see Table 2). For example, lower limb strengthening [32] or psychosocial factors (such as outcome expectations, attention and monitoring, the interest and empathy expressed by physiotherapists and the credibility of the intervention) may contribute to improvements in pain and function $[33,34]$.

There is also a separate or further explanation for the null findings. Measuring change in physical activity using the self-report PASE involves a number of 
limitations that may increase the chance of a Type II error (i.e. rejecting an association between change in physical activity and clinical outcome if one exists). Although the PASE has been highlighted as a promising measure of physical activity in older adults with OA [35], all self-report measures of physical activity can either over- or under- estimate actual physical activity level [36] since they are at risk of recall bias (through errors in memory and activity recall), misclassification of physical activity intensity and duration $[15,37]$, and social desirability bias by participants [38]. Furthermore, modelling change in physical activity is methodologically challenging and using an absolute change score between two timepoints may compound measurement errors and reduce regression coefficient precision, biasing our findings towards the null [39].

The PASE minimal detectable change statistic (87), which measures the threshold for a "real" change that with 95\% confidence is beyond measurement error [40], is considerably larger than the mean change detected in the BEEP dataset (15.1). This suggests that the mean change in PASE scores detected during the exercise interventions was relatively small, potentially affected by measurement error and perhaps insufficient to influence clinical outcomes or alternatively that the PASE may have inadequate responsiveness in older adults with joint pain.

To the authors' knowledge, this is the first study to explicitly investigate the association between change in physical activity level and clinical outcomes of pain and function in older adults with knee pain attributable to OA. However, similar studies have investigated the association between change in physical activity level and disability in those with low back pain [41] and pain severity and physical function outcomes in those with fibromyalgia $[42,43]$. Similar to our findings, no association was found in the back pain study using either self-report or accelerometer measured physical activity [41], however, associations were found between change in physical activity and future clinical outcomes in the two fibromyalgia studies [42, 43]. Whilst the aetiological differences between knee pain attributed to $\mathrm{OA}$ and fibromyalgia are likely to be substantial and prevent direct comparison, these findings do suggest it is possible to detect associations if they exist between self-report change in physical activity and clinical outcomes despite the previously discussed challenges in measuring change in physical activity.

\section{Strengths and limitations}

The strengths of this study were the ability to investigate both univariable and adjusted associations between change in physical activity and future clinical outcomes at two separate time-points. The use of multiple imputation helped preserve sample size, reducing the risk of bias due to loss to follow-up [29] whilst the sensitivity analysis added strength to the primary findings by exploring the dataset under different missing data assumptions.

The main study limitation, relating to the challenges in measuring change in physical activity, has been discussed above. With our available data we were unable to investigate levels of different types of physical activity, for example, time spent in strengthening or different cardiovascular intensities of exercise. Different types of physical activity may have had different effects on outcome. Another concern for our analysis is temporal bias. Temporal bias occurs when the inference about proper temporal sequence of cause and effect is erroneous [44]. Attempts were made to measure the exposure of interest- change in physical activity level (baseline to three months) prior to the clinical outcomes (at three and six months). Nevertheless, change in pain or physical function may have occurred prior to any change in physical activity, meaning we cannot be sure about the direction of any potential cause and effect. In handling missing data using multiple imputation for our analysis, we made the assumption that our data was "missing at random" (MAR) [29] since we deemed it likely that missing values could be estimated from observed values. If any missing data was "missing not at random" (MNAR) i.e. there are systematic differences between missing and observed values even after the observed data are taken into account then our multiple imputed analysis would be at risk of bias [29].

Finally, before generalising our findings it is important to remember this sample population were older adults with knee pain attributed to clinically diagnosed OA from a RCT of exercise interventions. Other populations of older adults with knee pain, for example, those who did not consent to exercise interventions may have different clinical outcomes with changing their physical activity levels.

\section{Implications}

Although change in physical activity was not shown to be associated with clinical outcomes of pain and physical function, insufficiently active older adults with knee pain can still be advised to increase their physical activity levels as able, in order to achieve the associated general health benefits [45-48] with the reassurance that modest increases in physical activity are not associated with increasing pain or deterioration in function at a group level.

In order to select the most appropriate physical activity measure for longitudinal studies measuring change in physical activity in older adults with knee pain, future research could investigate and compare the reliability and responsiveness of the PASE alongside other recommended measures of physical activity such as the International Physical Activity Questionnaire (IPAQ) [37] and direct measures such as pedometry and accelerometry. 
The inclusion criteria for this trial sample was based on older adults with knee pain regardless of baseline level of physical activity. Future studies could investigate specific subgroups of older adults with knee pain, such as those who are inactive, who may plausibly respond differently to increases in physical activity than all adults with knee pain or those currently meeting guideline recommended activity levels.

\section{Conclusions}

Change in physical activity level was not associated with future pain, physical function or the proportion of participants who could be classified as treatment responders in this secondary analysis of a randomised trial dataset. Hence other factors may be responsible for improvements in these clinical outcomes following exercise interventions. Accurately measuring change in physical activity in older adults with knee pain remains a challenge and the PASE, although useful for capturing population snap shots of physical activity level, may not be sufficiently responsive to detect changes over time in this clinical population. We recommend the further investigation of responsiveness in commonly utilised measures of physical activity for older adults with joint pain including the PASE to aid future longitudinal studies assessing change in physical activity.

\section{Additional files}

Additional file 1: Table S1. Summary of the BEEP trial interventions (Foster et al. [12]). (DOCX $15 \mathrm{~kb}$ )

Additional file 2 Supplementary material: Adjusted model building detail. (DOCX $15 \mathrm{~kb}$ )

\section{Abbreviations}

BEEP: Benefits of Effective Exercise for Knee Pain; BMI: Body Mass Index; GAD7: Generalised Anxiety Disorder-7; IPAQ: International Physical Activity Questionnaire; ISRCTN: International Standard Registered Clinical/soCial sTudy Number; ITE: Individually Tailored Exercise; MDC: Minimal Detectable Change; NIHR: National Institute for Health Research; OA: osteoarthritis; OMERACTOARSI: Outcome Measures in Rheumatology Clinical Trials-Osteoarthritis Research Society International; OR: odds ratio; PA: Physical activity; PASE: Physical Activity Scale for the Elderly; PHQ 8: Personal Health Questionnaire 8; RCT: Randomised controlled trial; REC: Research Ethics Committee; TEA: Targeted Exercise Adherence; UC: Usual Care; WOMAC: Western Ontario and McMaster Universities Osteoarthritis Index

\section{Acknowledgments}

We would like to thank all the participants and staff involved with the BEEP trial, including Elaine Nicholls for data cleaning of the BEEP dataset and statistical input with the multiple imputation of missing data.

\section{Funding}

JQ was funded for this work by a Keele University Acorn PhD studentship. He is now funded by a National Institute for Health Research (NIHR) Academic Clinical Lectureship in Physiotherapy, awarded as part of Professor Christian Mallen's NIHR Research Professorship (NIHR-RP-2014-026). Neither funder had involvement in the study design, collection, data analysis, writing or publishing of this paper.

NF was supported through an NIHR Research Professorship (NIHR-RP-011-015) and is a NIHR Senior Investigator.
MH was supported through an NIHR School for Primary Care Research postdoctoral fellowship.

The views expressed in this publication are those of the authors and not necessarily those of the NHS, the NIHR or the Department of Health.

\section{Availability of data and materials}

The datasets used and/or analysed during the current study are available from the corresponding author on reasonable request.

\section{Authors' contributions}

$J Q$ was the overall lead for the work and was involved at all stages of the paper. JQ, NF, PC, RO and MH were involved with the conception of the design. JQ and $\mathrm{RO}$ were involved in data analysis. All authors were involved in the interpretation of the data, drafting of the article and final approval of the submitted version.

\section{Authors' information}

The lead author can be contacted by email.j.g.quicke@keele.ac.uk or at Primary Care and Health Sciences, David Wetherall Building, Keele University, Keele, Staffordshire, United Kingdom, ST5 5BG.

Ethics approval and consent to participate

The trial received research ethical approved by the North West 1 Research Ethics Committee, Cheshire, UK (REC reference:10/H1017/45). All study participants gave full informed written consented to take part [12].

\section{Consent for publication}

All authors provided their consent for this publication.

\section{Competing interests}

The authors declare that they have no competing interests.

\section{Publisher's Note}

Springer Nature remains neutral with regard to jurisdictional claims in published maps and institutional affiliations.

Received: 30 September 2017 Accepted: 6 February 2018

Published online: 17 February 2018

\section{References}

1. National Institute for Health and Care Excellence. Osteoarthritis: care and management in adults. NICE guideline 177, London. 2014.

2. Fernandes L, Hagen KB, Bijlsma JWJ, Andreassen O, Christensen P, Conaghan PG, et al. EULAR recommendations for the non-pharmacological core management of hip and knee osteoarthritis. Ann Rheum Dis. 2013; 72(7):1125-35.

3. McAlindon TE, Bannuru RR, Sullivan MC, Arden NK, Berenbaum F, BiermaZeinstra SM, et al. OARSI guidelines for the non-surgical management of knee osteoarthritis. Osteoarthr Cartil. 2014;22(3):363-88.

4. Uthman OA, van der Windt DA, Jordan JL, Dziedzic KS, Healey EL, Peat GM, et al. Exercise for lower limb osteoarthritis: systematic review incorporating trial sequential analysis and network meta-analysis. Br Med J. 2013;347:f5555.

5. Juhl C, Christensen R, Roos EM, Zhang W, Lund H. Impact of exercise type and dose on pain and disability in knee osteoarthritis: a systematic review and meta-regression analysis of randomized controlled trials. Arthritis Rheumatology. 2014;66(3):622-36.

6. Fransen M, McConnell S, Harmer AR, Van der Esch M, Simic M, Bennell KL. Exercise for osteoarthritis of the knee: a Cochrane systematic review. British Journal of Sports Medicine. 2015:bjsports - 2015-095424.

7. Runhaar J, Luijsterburg P, Dekker J, Bierma-Zeinstra SMA. Identifying potential working mechanisms behind the positive effects of exercise therapy on pain and function in osteoarthritis; a systematic review. Osteoarthr Cartil. 2015;23(7):1071-82.

8. Stubbs B, Hurley M, Smith T. What are the factors that influence physical activity participation in adults with knee and hip osteoarthritis? A systematic review of physical activity correlates. Clin Rehabil. 2015:29(1):80-94.

9. Pellegrini CA, Song J, Chang RW, Semanik PA, Lee J, Ehrlich-Jones L, Pinto D, Dunlop DD. Change in physical activity and sedentary time associated with 2-year weight loss in obese adults with osteoarthritis. J Phys Act Health. 2016;13(5):461-6.

10. Messier SP, Mihalko SL, Legault C, Miller GD, Nicklas BJ, DeVita P, et al. Effects of intensive diet and exercise on knee joint loads, inflammation, and 
clinical outcomes among overweight and obese adults with knee osteoarthritis: the IDEA randomized clinical trial. JAMA. 2013;310(12):1263.

11. Pham T, Van Der Heijde D, Lassere M, Altman RD, Anderson JJ, Bellamy N, et al. Outcome variables for osteoarthritis clinical trials: the OMERACT-OARSI set of responder criteria. J Rheumatol. 2003;30(7):1648-54.

12. Foster NE, Healey EL, Holden MA, Nicholls E, Whitehurst DG, Jowett $\mathrm{S}$, et al. A multicentre, pragmatic, parallel group, randomised controlled trial to compare the clinical and cost-effectiveness of three physiotherapy-led exercise interventions for knee osteoarthritis in older adults: the BEEP trial protocol (ISRCTN: 93634563). BMC Musculoskelet Disordors. 2014;15(1):254

13. Bellamy N, Buchanan WW, Goldsmith CH, Campbell J, Stitt LW. Validation study of WOMAC: a health status instrument for measuring clinically important patient relevant outcomes to antirheumatic drug therapy in patients with osteoarthritis of the hip or knee. J Rheumatol. 1988 Dec;15(12):1833-40.

14. McConnell S, Kolopack P, Davis AM. The western Ontario and McMaster universities osteoarthritis index (WOMAC): a review of its utility and measurement properties. Arthritis Rheumatology. 2001:45(5):453-61.

15. Trudeau J, Van Inwegen R, Eaton T, Bhat G, Paillard F, Ng D, et al. Assessment of pain and activity using an electronic pain diary and actigraphy device in a randomized, placebo-controlled crossover trial of celecoxib in osteoarthritis of the knee. Pain Practice. 2015:15(3):247-55.

16. Pham T, van der Heijde D, Altman RD, Anderson JJ, Bellamy N, Hochberg M, et al. OMERACT-OARSI initiative: osteoarthritis research society international set of responder criteria for osteoarthritis clinical trials revisited. Osteoarthr Cartil. 2004;12(5):389-99.

17. Washburn RA, Smith KW, Jette AM, Janney CA. The physical activity scale for the elderly (PASE): development and evaluation. J Clin Epidemiol. 1993; 46(2):153-62.

18. Martin KA, Rejeski WJ, Miller ME, James MK, Ettinger Jr. WH, Messier SP. Validation of the PASE in older adults with knee pain and physical disability. Med Sci Sports Exerc 1999;31(5):627-633.

19. Sharma L, Cahue S, Song J, Hayes K, Pai YC, Dunlop D. Physical functioning over three years in knee osteoarthritis: role of psychosocial, local mechanical, and neuromuscular factors. Arthritis Rheum. 2003:48(12):3359-70.

20. Dunlop DD, Song J, Semanik PA, Sharma L, Chang RW. Physical activity levels and functional performance in the osteoarthritis initiative: a graded relationship. Arthritis Rheum. 2011;63(1):127-36.

21. Fransen M, Su S, Harmer A, Blyth FM, Naganathan V, Sambrook $P$, et al. A longitudinal study of knee pain in older men: concord health and ageing in men project. Age Ageing. 2014;43(2):206-12.

22. Cleveland RJ, Luong M-LN, Knight JB, Schoster B, Renner JB, Jordan JM, et al. Independent associations of socioeconomic factors with disability and pain in adults with knee osteoarthritis. BMC Musculoskelet Disorders. 2013:14:297.

23. Neogi T, Zhang Y. Epidemiology Of OA. Rheum Dis Clin N Am. 2013;39(1):1-19.

24. Office for National Statistics. Standard Occupational Classification 2010 (volume 3).

25. Kroenke K, Spitzer RL, Williams JB. The PHQ-9: validity of a brief depression severity measure. J Gen Intern Med. 2001;16(9):606-13.

26. Spitzer RL, Kroenke K, Williams JBW, Löwe B. A brief measure for assessing generalized anxiety disorder: the GAD-7. Arch Intern Med. 2006;166(10):1092-7.

27. MacFarlane GJ, Croft PR, Schollum J, Silman AJ. Widespread pain: is an improved classification possible? J Rheumatol. 1996;23(9):1628-32.

28. StataCorp. Stata statistical software: release 13. College Station, TX: StataCorp LP; 2013.

29. Sterne JAC, White IR, Carlin JB, Spratt M, Royston P, Kenward MG, et al. Multiple imputation for missing data in epidemiological and clinical research: potential and pitfalls. Br Med J. 2009;338:b2393.

30. Hay EM, Dziedzic K, Foster NE, Peat G, van der Windt DA, Bartlam B...Croft, P. Clinical osteoarthritis and joint pain in older people: optimal management in primary care. NIHR Programme grant for Applied Research final report, RP-PG-0407-10386. (in press).

31. Tariss TWA. Primer in longitudinal data analysis. London: SAGE Publications; 2000.

32. Knoop J, Steultjens MPM, Roorda LD, Lems WF, van der Esch M, Thorstensson CA, et al. Improvement in upper leg muscle strength underlies beneficial effects of exercise therapy in knee osteoarthritis: secondary analysis from a randomised controlled trial. Physiotherapy. 2014; 101(2):171-7.

33. Hall AM, Ferreira PH, Maher CG, Latimer J, Ferreira ML. The influence of the therapist-patient relationship on treatment outcome in physical rehabilitation: a systematic review. Phys Ther. 2010;90(8):1099-110.

34. Bennell $\mathrm{KL}$, Kyriakides $\mathrm{M}$, Hodges PW, Hinman RS. Effects of two physiotherapy booster sessions on outcomes with home exercise in people with knee osteoarthritis: a randomized controlled trial. Arthritis Care Res. 2014:66(11):1680-7.

35. Terwee CB, Bouwmeester W, van Elsland SL, de Vet HCW, Dekker J. Instruments to assess physical activity in patients with osteoarthritis of the hip or knee: a systematic review of measurement properties. Osteoarthr Cartil. 2011;19(6):620-33.

36. Prince SA, Adamo KB, Hamel ME, Hardt J, Gorber SC, Tremblay MA. Comparison of direct versus self-report measures for assessing physical activity in adults: a systematic review. The International Journal of Behavioral Nutrition and Physical Activity. 2008;5:56.

37. Craig CL, Marshall AL, Sjöström M, Bauman AE, Booth ML, Ainsworth BE, et al. International physical activity questionnaire: 12-country reliability and validity. Med Sci Sports Exerc. 2003;35(8):1381-95.

38. Adams SA, Matthews CE, Ebbeling CB, Moore CG, Cunningham JE, Fulton J, et al. The effect of social desirability and social approval on self-reports of physical activity. Am J Epidemiol. 2005;161(4):389-98.

39. Polit DF, Yang F. Measurement and the measurement of change. London: Wolters Kluwer; 2015.

40. Svege I, Kolle E, Risberg M. Reliability and validity of the physical activity scale for the elderly (PASE) in patients with hip osteoarthritis. BMC Musculoskelet Disord. 2012;13(1):26.

41. Hendrick P, Milosavljevic S, Hale L, Hurley DA, McDonough SM, Herbison P, et al. Does a patient's physical activity predict recovery from an episode of acute low back pain? A prospective cohort study. BMC Musculoskelet Disorders. 2013;14:126

42. Kaleth AS, Saha CK, Jensen MP, Slaven JE, Ang DC. Effect of moderate to vigorous physical activity on long-term clinical outcomes and pain severity in fibromyalgia. Arthritis Care and Research. 2013;65(8):1211-8.

43. Steiner JL, Bigatti SM, Ang DC. Trajectory of change in pain, depression, and physical functioning after physical activity adoption in fibromyalgia. J Health Psychol. 2015;20(7):931-41.

44. Szklo M, Nieto FJ. Epidemiology: Beyond the Basics. Jones \& Bartlett Publishers; 2014

45. Warburton D, Nicol CW, Bredin SSD. Health benefits of physical activity: the evidence. Can Med Assoc J. 2006:174(6):801-9.

46. Chodzko-Zajko WJ, Proctor DN, Fiatarone Singh MA, Minson CT, Nigg CR, Salem GJ, et al. Exercise and physical activity for older adults. Med Sci Sports Exerc. 2009;41(7):1510-30.

47. Department of Health. Start Active, Stay Active. 2011;1-62.

48. Autenrieth CS, Kirchberger I, Heier M, Zimmermann A-K, Peters A, Döring A, et al. Physical activity is inversely associated with multimorbidity in elderly men: results from the KORA-age Augsburg study. Prev Med. 2013;57(1):17-9.

\section{Submit your next manuscript to BioMed Central and we will help you at every step:}

- We accept pre-submission inquiries

- Our selector tool helps you to find the most relevant journal

- We provide round the clock customer support

- Convenient online submission

- Thorough peer review

- Inclusion in PubMed and all major indexing services

- Maximum visibility for your research

Submit your manuscript at www.biomedcentral.com/submit
) Biomed Central 